\title{
ARTICLE
}

Clinical Research

\section{Weight loss normalizes enhanced expression of the oncogene survivin in visceral adipose tissue and blood leukocytes from individuals with obesity}

\author{
Andrea G. Izquierdo $\mathbb{1}^{1,2} \cdot$ Marcos C. Carreira ${ }^{2,3}$ - Gemma Rodriguez-Carnero ${ }^{1}$ - Alfredo Fernandez-Quintela ${ }^{2,4}$.

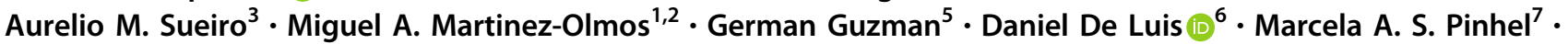 \\ Carolina F. Nicoletti ${ }^{7}$ - Carla B. Nonino ${ }^{7}$. Francisco J. Ortega $\mathbb{D}^{2,8} \cdot$ Maria P. Portillo $^{2,4} \cdot$ Jose M. Fernandez-Real $^{2,8}$. \\ Felipe F. Casanueva ${ }^{2,3}$. Ana B. Crujeiras $\mathbb{1 D}^{1,2}$
}

Received: 18 February 2020 / Revised: 21 May 2020 / Accepted: 1 June 2020 / Published online: 16 June 2020

(c) The Author(s), under exclusive licence to Springer Nature Limited 2020

\begin{abstract}
Background/objectives Survivin is an oncogene associated with a decrease in apoptosis, an increase in tumor growth, and poor clinical outcome of diverse malignancies. A correlation between obesity, cancer, and survivin is reported in the literature. To date, the impact of weight loss on change in survivin levels is understudied. This study was aimed at: (1) comparing survivin levels in adipose tissue (AT) from lean and obese animal models and evaluating changes after weight loss induced by energy restriction and/or exercise; (2) comparing survivin levels in normal weighted and obese humans and evaluating changes in survivin levels after weight loss induced by a very-low-calorie ketogenic diet (VLCKD) or bariatric surgery in AT and/or blood leukocytes (PBL/PBMCs).

Subjects/methods Survivin expression was evaluated in subcutaneous (SAT) and visceral (VAT) AT derived from animal models of monogenic (Zucker rats) and diet-induced obesity (Sprague Dawley rats and C57BL/6J mice) and after a 4-week weight-loss protocol of energy restriction and/or exercise. Plasma was used to measure the inflammatory status. Survivin expression was also evaluated in PBMCs from patients with obesity and compared with normal weight, in PBLs after VLCKD, and in SAT and/or PBLs after bariatric surgery.

Results Survivin expression was specifically higher in VAT from obese that lean animals, without differences in SAT. It decreased after weight loss induced by energy restriction and correlated with adiposity and inflammatory markers. In humans, the correlation between being obese and higher levels of survivin was confirmed. In obese subjects, survivin levels were reduced following weight loss after either VLCKD or bariatric surgery. Particularly, a decrease in PBMCs expression (not in SAT one) was found after surgery.

Conclusions Weight loss is effective in decreasing survivin levels. Also, PBL/PBMC should be regarded as appropriate mirror of survivin levels in VAT for the identification of an obesity-related protumoral microenvironment.
\end{abstract}

These authors contributed equally: Andrea G. Izquierdo, Marcos C. Carreira

Supplementary information The online version of this article (https:// doi.org/10.1038/s41366-020-0630-7) contains supplementary material, which is available to authorized users.

Ana B. Crujeiras

anabelencrujeiras@hotmail.com

Extended author information available on the last page of the article

\section{Introduction}

Obesity, a disease with epidemic proportions [1-3], is responsible for the development of numerous complications such as type 2 diabetes [4, 5], cardiovascular diseases [6], and several types of cancer [7]. This disease originates as a result of a positive energy balance that triggers an abnormal expansion and dysfunction of white adipose tissue $[8,9]$ and secretion of several bioactive molecules such as adipokines [10]. In addition, this excessive accumulation of visceral and ectopic fat leads to chronic low-grade inflammation [11], so recent research postulates the dysfunction of visceral adipose tissue (VAT) characteristic of obesity as the 
Table 1 Characteristics of animal experimental groups (Zucker rats, Sprague Dawley-DIO rats, and C57BL/6J-DIO mice).

\begin{tabular}{|c|c|c|c|c|c|c|c|c|c|c|}
\hline & \multicolumn{5}{|l|}{ Zucker rats } & \multicolumn{2}{|l|}{ DIO rats } & \multicolumn{3}{|l|}{ DIO mice } \\
\hline & Lean & $\mathrm{Ob} \mathrm{AL}$ & Ob ER & Ob EX & Ob EREX & Lean & DIO & Lean & DIO & $\mathrm{CR}$ \\
\hline$n$ & 10 & 10 & 10 & 10 & 10 & 12 & 14 & 4 & 8 & 8 \\
\hline Age (weeks) & 20 & 20 & 20 & 20 & 20 & 16 & 16 & 38 & 38 & 38 \\
\hline Body weight (kg) & $428.4 \pm 20.2$ & $617.7 \pm 46.3^{\mathrm{a}}$ & $462.2 \pm 38.5^{\mathrm{b}}$ & $606.3 \pm 44.8^{\mathrm{a}, \mathrm{c}}$ & $455.3 \pm 37.6^{\mathrm{b}, \mathrm{d}}$ & $228.5 \pm 12.0$ & $243.5 \pm 21.5^{\mathrm{a}}$ & $34.5 \pm 2.7$ & $53.0 \pm 2.7^{\mathrm{a}, \mathrm{c}}$ & $42.5 \pm 5.2^{\mathrm{a}, \mathrm{b}}$ \\
\hline Fat mass $(\mathrm{g})$ & $36.7 \pm 7.4$ & $252.2 \pm 24.8^{\mathrm{a}}$ & $184.5 \pm 26.3^{\mathrm{a}, \mathrm{b}}$ & $245.3 \pm 18.9^{\mathrm{a}, \mathrm{c}}$ & $173.6 \pm 19.1^{\mathrm{a}, \mathrm{b}, \mathrm{d}}$ & $15.6 \pm 5.2$ & $32.5 \pm 5.7^{\mathrm{a}}$ & - & - & - \\
\hline Fat-free mass (g) & $321.2 \pm 13.6$ & $296.5 \pm 17.7$ & $241.1 \pm 12.0^{\mathrm{a}, \mathrm{b}}$ & $292.9 \pm 21.6^{\mathrm{c}}$ & $246.0 \pm 18.5^{\mathrm{a}, \mathrm{b}, \mathrm{d}}$ & $172.3 \pm 7.8$ & $170.0 \pm 16.1$ & - & - & - \\
\hline
\end{tabular}

$O b A L$ obese ad libitum, $O b E R$ obese energy restriction, $O b E X$ obese exercise, $O b E R E X$ obese energy restriction and exercise, $D I O$ diet-induced obesity, $C R$ caloric restriction.

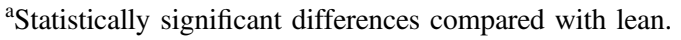

${ }^{\mathrm{b}}$ Statistically significant differences compared with $\mathrm{Ob} \mathrm{AL}$ or DIO.

${ }^{\mathrm{c}}$ Statistically significant differences compared with Ob ER or CR.

${ }^{\mathrm{d}}$ Statistically significant differences compared with Ob EX.

mechanism responsible for the development of associated complications, such as metabolic diseases or cancer $[12,13]$. In this line, previous works demonstrate that the link between obesity and cancer may be associated with the microenvironment generated by the effect of VAT dysfunction, which could promote the development of tumor [14]. In fact, waist-related measures were predictors of obesity-related cancer in a gender-dependent manner [15]. The cellular mechanisms underlying the association between obesity and cancer are not yet fully known, so new findings in this field will be very relevant in personalized and precision medicine for the treatment of cancers associated with obesity.

The oncogene survivin is a key member of the family of apoptosis inhibitors and is involved in the promotion of cell division. Survivin expression is increased in most tumors [16-19] and it may affect the response of tumor cells to anticancer agents and may be associated with alterations in survival in carcinogenic processes [19-22]. Survivin also acts on several types of non-tumor cells and tumor-free tissues [23], such as the fraction of AT-derived stem cells of obese subjects [24], and it has been identified as a key regulator of lipid storage and adipocyte metabolism [25]. Altered expression of survivin in non-tumor breast tissue of rats [26], in human cell line of non-tumor breast [26], and in tumor-free liver in rats [27] was also found, all in association with the effect of factors secreted by AT in obesity. These effects were also reflected in the circulating leukocytes of women with obesity with breast cancer and without tumor [26].

This work was aimed to evaluate and compare the expression of survivin in subcutaneous adipose tissue (SAT) and VAT of animal models of tumor-free obesity and determine the possible effect of weight-loss interventions. In addition, we aim to validate these results in patients with obesity and after therapies for weight loss (very-low-calorie ketogenic diet (VLCKD) [28] and bariatric surgery).

\section{Materials and methods}

\section{Animals}

\section{Monogenic obesity}

The experimental studies (Table 1) were performed using 8week-old male obese Zucker rats (fa/fa, $n=40)$ and lean Zucker rats $(F a / f a, n=10)$ that were purchased from Charles River Laboratories (Barcelona, Spain). They were allowed to acclimatize for 1 week upon arrival and were provided with free access to water and a standard laboratory diet (SAFE-Panlab, Spain) that was comprised of 5.5\% lipid, 23\% protein, and 70\% carbohydrate content. Body weight (BW) and food and water intake were measured throughout the experimental period.

A group of obese rats $(f a l f a, n=30)$ was randomly divided into three subgroups that included an energy restriction group (Ob ER; $n=10)$, an exercise group (Ob $\mathrm{EX} ; n=10$ ), and an energy restriction plus exercise group (Ob EREX; $n=10$ ). These obese rats were individually housed for 1 week, and their individual food intake was weighed and recorded. Then, the rats in the Ob ER and the Ob EREX groups were fed a diet that was 30\% less in quantity compared with that of their individual food intake for 4 weeks (based on the weight of food), and the remaining rats and the rats in the control group (Ob $\mathrm{AL}$; $n=10$ ) were maintained on an ad libitum diet. Animals from the $\mathrm{Ob} \mathrm{EX}$ and the $\mathrm{Ob}$ EREX groups were placed on a monitored rodent treadmill (Treadmill system 303401-R04/C, TSE-Systems, Inc, USA) for $10 \mathrm{~min} /$ day, and the 
intensity of the exercise was increased progressively from $10 \mathrm{~m} / \mathrm{min}$ to $20 \mathrm{~m} / \mathrm{min}$ for 1 week to allow for familiarization. The animals were then placed on the treadmill for $30 \mathrm{~min} /$ day at $20 \mathrm{~m} / \mathrm{min}$, and this was continued for 7 days per week over a period of 4 weeks.

\section{Diet-induced obesity (DIO)}

Twenty-six male, 3-week old Sprague Dawley rats, and 20 male, 8-week old C57BL/6J mice [29], were obtained from the central animal facilities of the Universidad de Santiago de Compostela. After the acclimatization period (1 week), rodents were randomized into weight-matched groups (two groups and $n=12-14$ animals/group in rats; three groups and $n=4-8$ animals/group in mice). One group was provided with ad libitum access to a standard diet (SAFEPanlab, Spain). The other groups were fed with a high-fat diet (HFD) (Open Source Diets, Research Diets; Brogaarden, Denmark, Reference D 12492) that consisted of $60 \%$ lipid, $20 \%$ protein, and $20 \%$ carbohydrate for 12 weeks in rats and 29 weeks in mice and, in addition, the group caloric restriction (CR) of mice was fed a diet that was $30 \%$ less in quantity compared with that of their individual food intake for last 3 weeks (based on the weight of food). BW, food, and water intake were measured throughout the experimental period (Table 1).

All animal experiments and procedures used in this study were approved by the Animal Care Committee of the University of Santiago de Compostela in accordance with our institutional guidelines and the European Union standards for the care and use of experimental animals.

\section{Humans}

\section{Obese and normal-weight patients as a cross-sectional cohort}

A group of healthy humans exhibiting normal weight $(n=$ $29 ; 35.8 \pm 9.4$ years, $\left.22.1 \pm 2.2 \mathrm{~kg} / \mathrm{m}^{2}\right)$ or obesity $(n=22$; $40.1 \pm 11.3$ years; $39.1 \pm 9.2 \mathrm{~kg} / \mathrm{m}^{2}$ ) was recruited for our study (Table 2). All participants were in apparent good health according to their medical history, physical examination, and routine biochemical and hematologic laboratory test results. The participants reported no use of supplemental vitamins, minerals, or regular prescription medications during the previous 3 months. Blood samples were obtained after overnight fasting, and the plasma was separated by centrifugation at $1268 \times g$ and $4{ }^{\circ} \mathrm{C}$ for $15 \mathrm{~min}$; then was frozen at $-80^{\circ} \mathrm{C}$ for subsequent analyses. The PBMCs were isolated by differential centrifugation using Polymorphprep (Axis Shield PoC AS, Norway). The cell pellet was resuspended in Trizol reagent (Invitrogen,

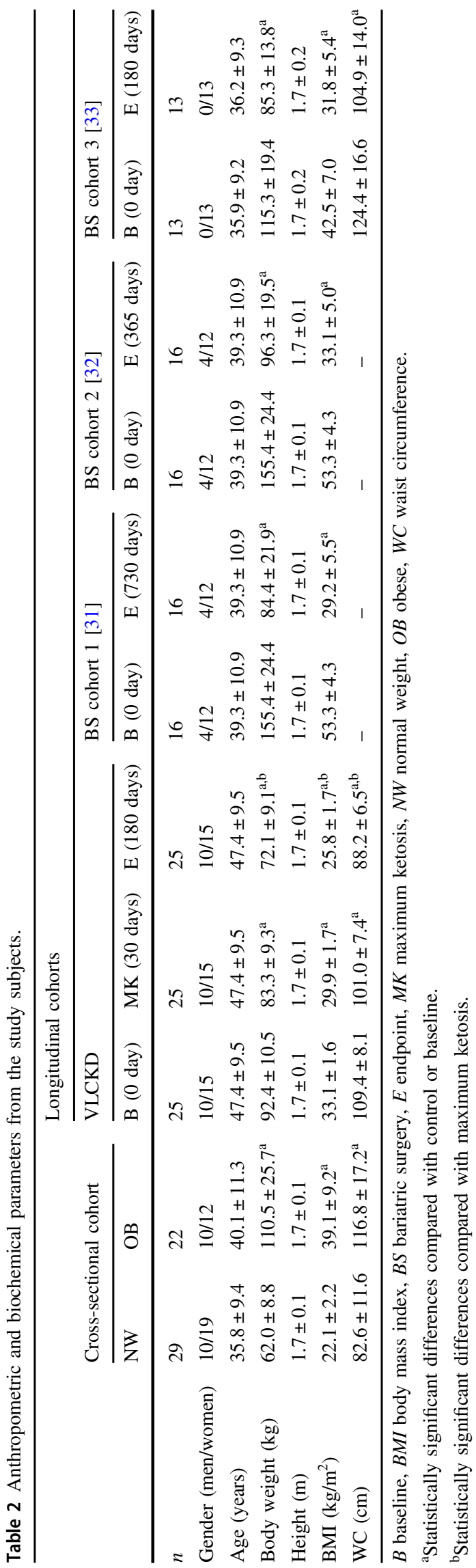


Carlsbad, USA) and immediately frozen at $-80{ }^{\circ} \mathrm{C}$ until the RNA was extracted.

\section{Obese patients following a VLCKD to lose weight as a longitudinal cohort}

A group of obese patients undergoing treatment at the Department of Endocrinology and Nutrition at the University Clinical Hospital of Valladolid was recruited to undergo a nutritional intervention based on a VLCKD as a treatment for weight loss (PronoKal Method) [30, 31]. The range of ages and body mass indexes (BMIs) of the obese patients were $1-65$ years and 30 to $35 \mathrm{~kg} / \mathrm{m}^{2}$, respectively. All participants $(n=25)$ were generally healthy individuals, and they exhibited no serious medical conditions (Table 2). The study consisted of a clinical trial that possessed an open and controlled design and included an evaluation by a physician and expert dietitian, group meetings, and exercise recommendations performed in a hospital setting at 0 (baseline), 30 (maximum ketosis), and 180 (endpoint) days from the start of treatment (clinicaltrial.gov: NCT01865448). Blood samples were obtained after overnight fasting, and the plasma was separated by centrifugation at $1268 \times g$ and $4{ }^{\circ} \mathrm{C}$ for $15 \mathrm{~min}$. The plasma samples were then frozen at $-80^{\circ} \mathrm{C}$ for subsequent analyses. PBL were stabilized in RNAlater Solution (Invitrogen, Carlsbad, USA) and immediately frozen at $-80^{\circ} \mathrm{C}$ until the RNA was extracted.

\section{Obese patients following a bariatric surgery as additional longitudinal cohorts}

The cohorts 1 and 2 were constituted by samples of SAT from morbidly obese women (BMI $>40 \mathrm{~kg} / \mathrm{m}^{2}, n=16$ ) before and $\sim 1$ and $\sim 2$ years after surgery-induced weight loss (Roux-en-Y gastric bypass (RYGB)). All these subjects were recruited at the Endocrinology Service of the Hospital of Girona "Dr Josep Trueta," were of Caucasian origin, and reported that their BW had been stable for at least 3 months before the study. Patients had no systemic disease other than type 2 diabetes and obesity and all were free of any infections in the previous month before the study. Samples and data from patients were partially provided by the FATBANK platform promoted by the CIBEROBN and coordinated by the IDIBGI Biobank. The average BMI of bariatric patients was reduced by more than $30 \%$ after surgery (Table 2).

Wide gene expression (Affymetrix GeneChip ${ }^{\circledR}$ Human Gene 2.0 ST Array) and miRNA (miRNA 3.0 Array) profiles were analyzed by Ortega et al. [32]. Global gene expression using two different microarray platforms $(\mathrm{AB}$ 1700 and Illumina) was analyzed by Dankel et al. [33].

The cohort 3 was constituted by samples of PBMCs from women with morbid obesity (BMI $>40 \mathrm{~kg} / \mathrm{m}^{2}, n=13$ ) before and 6 months after RYGB. All subjects were recruited at the Hospital das Clínicas of the Ribeirão Preto Medical School from Brazil. Patients with thyroid disease, cancer, and psychiatric disorders were excluded. After surgery, the average BMI of the patients was reduced by $25 \%$ (Table 2). Whole gene expression profiles were determined by microarray analysis (Illumina BeadCheap HumanHT-12_v4 Array) [34].

All participants of the studies provided written informed consent validated and approved by the Ethics Committee of the institution (CEIC Galicia No. 2009/076; C.I: 40/13, PNK-DHA2013-01; CEIC Girona No. 223/2004; CAAE: 18973913.0.0000.5440).

\section{Body composition and biochemical measurements}

In the animal models, weekly body composition studies were assessed using an Echo Magnetic Resonance Imaging System (EchoMRI 700; Echo Medical System, USA). Rodents were euthanized with $\mathrm{CO}_{2}$ and decapitated, and organs and tissues were obtained, immediately frozen on dry ice, and stored at $-80{ }^{\circ} \mathrm{C}$ until analysis. Plasma levels of pro-inflammatory cytokine monocyte chemoattractant protein-1 (MCP-1) in Zucker rats were analyzed by ELISA following the manufacturer's protocol (Bio-Plex Pro $^{\mathrm{TM}}$ Cytokine MCP-1, Bio-Rad Laboratories, USA).

Patients were weighed on a calibrated scale (Seca 200 scale, Medical Resources, EPI Inc OH, USA). BMI was calculated as BW in kg divided by the square of the body height in meters. Waist circumference was measured using a standard flexible nonelastic metric tape over the midpoint between the last rib and the iliac crest, while the patient was standing and exhaling.

\section{Gene expression assessment}

RNA was isolated using Trizol (Invitrogen) from the VAT and SAT samples of rats and from leukocytes derived from patients according to the manufacturer's recommendations. The expression of survivin was assessed using TaqMan real-time PCR as previously describe [14, 27]. For data analysis, gene expression levels were normalized GAPDH as internal control, and they were expressed as the average value for the control group according to the $2^{-\Delta \Delta \mathrm{Ct}}$ method. RT-qPCR experiments were performed in compliance with the Minimum Information for Publication of Quantitative Real-Time PCR Experiments guidelines (http:// www.rdml. org/miqe).

\section{Protein extraction and western blot analysis}

All the steps for extraction and preparation protein samples from tissue were carried out at $4{ }^{\circ} \mathrm{C}$ by following the 
protocol required as previously described [27]. The data are expressed as percentages corrected for GAPDH (arbitrary units).

\section{Statistical analysis}

The sample size of the current study was calculated to detect differences in BW in both animal and human cohorts. It was calculated to achieve a $\alpha=0.05$, and a power $(1-\beta)$ of $80 \%$. Normal distributions were assessed using the Kolmogorov-Smirnov test and the Shapiro-Wilk test. Differences in BW and body composition, gene expression, and MCP-1 levels between the groups comprising subjects with normal weight and obesity were evaluated by Student's $t$ test. In addition, differences between the weightloss treatment groups were evaluated by univariant ANOVA for the animal model and by repeatedmeasurements ANOVA for the longitudinal cohort of patients; both analysis of variance followed by the Bonferroni multiple comparison test. The association between the expression of survivin and body composition and MCP1 levels was evaluated by the rho Spearman test. All data are expressed as the mean \pm standard deviation in the tables and as the mean \pm standard error of the mean (SEM) in the figures. $P$ values $<0.05$ were considered statistically significant, and a $P$ value $\leq 0.1$ was considered to be indicative of a trend for significance. Statistical analysis was performed using SPSS 21.0 software (SPSS Inc, USA) for Windows XP (Microsoft, USA) and GraphPad Prism 6.01 software (GraphPad Software Inc, USA).

\section{Results}

\section{Survivin gene expression in AT of rodents with obesity comparing with lean and in response to weight-loss intervention}

In Zucker model, we fed rats a standard diet (ad libitum) while monitoring their BW gain and body composition. At the end of the experiment, the rats with obesity ( $f a / f a$ ) exhibited a higher BW and higher fat mass (FM) compared with that of their lean littermates $(F a / f a)$ (Table 1$)$, without statistically significant differences in fat-free mass (FFM). Regarding to the expression of survivin (Fig. 1), no statistically significant differences were observed in SAT between rats with obesity and lean. By contrast, higher levels of both gene (Fig. 1a) and protein (Fig. 1b) expression were observed in VAT from rats with obesity than lean. These differences were statistically significant.

After 4 weeks of weight loss, Ob ER and Ob EREX groups possessed a BW less than $\mathrm{Ob}$ AL group and were similar to lean control (Table 1); however, no differences were observed between the $\mathrm{BW}$ of the $\mathrm{Ob} \mathrm{EX}$ and the $\mathrm{Ob}$ AL groups. According to BW loss, the amount of FM was also reduced by a similar amount in Ob ER and Ob EREX groups, and there was a modest reduction in the amount of FFM (Table 1).

In the context of weight loss, survivin gene expression (Fig. 1c) and protein levels (Fig. 1d) in SAT were reduced more significantly in $\mathrm{Ob}$ ER group, while $\mathrm{Ob} \mathrm{EX}$ and $\mathrm{Ob}$ EREX groups exhibited no statistically important changes. In VAT, a relevant and statistically significant reduction was observed in Ob ER and Ob EREX groups compared with $\mathrm{Ob}$ AL group (Fig. 1e, f). Ob EX group exhibited an expression similar to $\mathrm{Ob} \mathrm{AL}$ group, where the survivin expression levels were significantly higher than in Ob ER and Ob EREX groups (Fig. 1e, f).

Moreover, correlation analysis revealed a positive association between the survivin expression levels in VAT and FM (Fig. 1g) and the circulating levels of MCP-1 (Fig. 1h). All the observed associations were statistically significant except between survivin expression in VAT and FFM as well as the expression in SAT and BW, FM, FFM, and MCP-1 (Supplementary 1b-f).

In DIO models, we found similar data to those observed in Zucker rats. Specifically, Sprague Dawley rats with obesity exhibited a higher survivin gene expression in VAT compared with lean rats (Supplementary 2a). Mice with obesity also exhibited a higher survivin gene expression in VAT, which was reduced to levels similar to lean group after CR by weight loss (Supplementary 2b). Interestingly, in this DIO model, survivin expression in SAT was also increased in the group with obesity and reduced after CR (Supplementary 2b).

\section{Survivin gene expression in human with obesity comparing with normal weight and in response to weight-loss intervention}

The analysis of the survivin gene expression in PBMCs of individuals with obesity revealed higher levels compared with those of normal-weight individuals, mirroring the results observed in the VAT of the animal model (Fig. 2a). Moreover, a positive correlation was observed between survivin expression in PBMCs and the BMI (Fig. 2b). It is also relevant that the analysis of the survivin gene expression in the PBLs of patients with obesity undergoing a VLCKD revealed a decrease at the maximum ketosis point (mean of $9 \mathrm{~kg}$ of BW loss) and endpoint phase (mean of $20 \mathrm{~kg}$ of BW loss) compared with baseline (Fig. 2c). These results are in agreement with those obtained from our animal model after energy restriction-induced weight loss and again a positive correlation was observed between survivin expression in PBLs and the BMI (Fig. 2d). Evolution of 
A

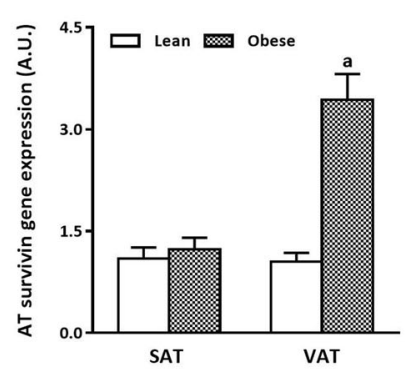

C

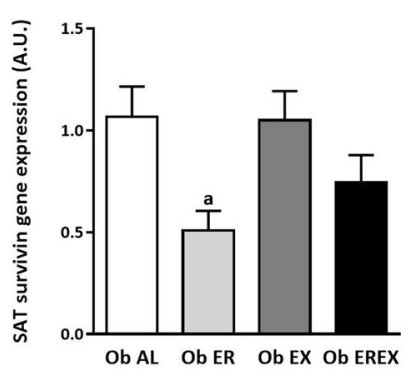

E

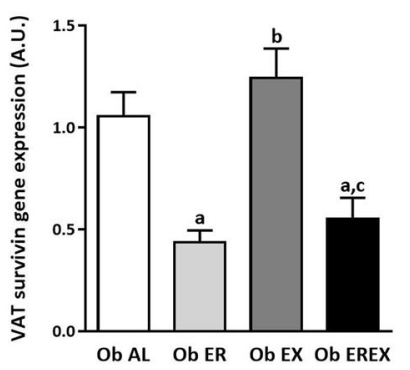

G

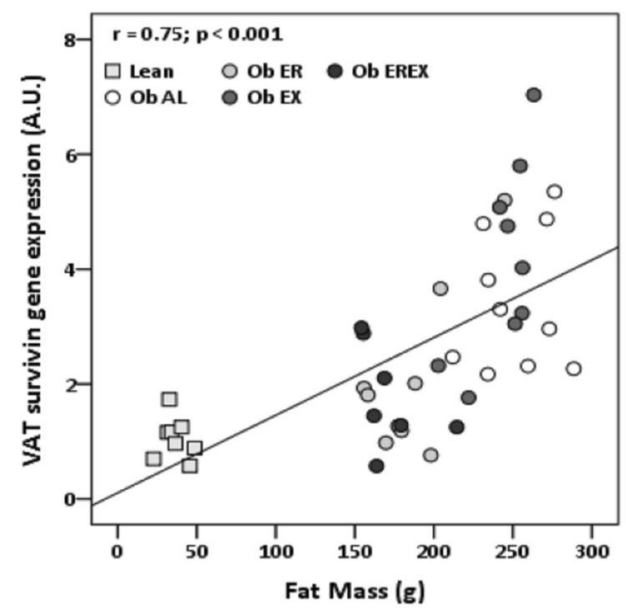

BW, waist circumference, and BMI is represented in Table 2.

To reinforce these findings, we analyzed previously published data from studies of bariatric surgery-induced
B
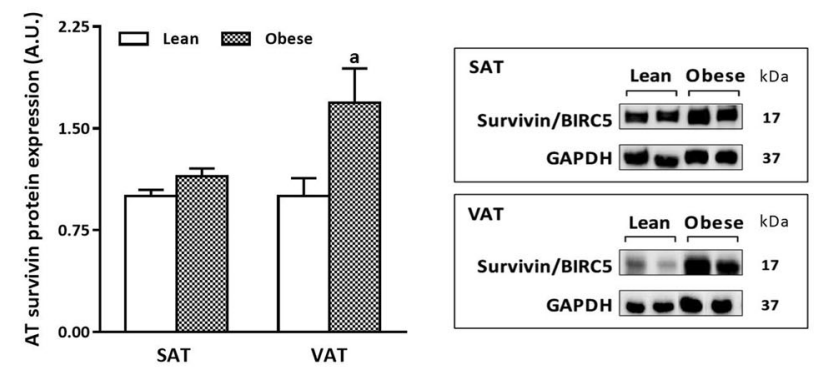

D
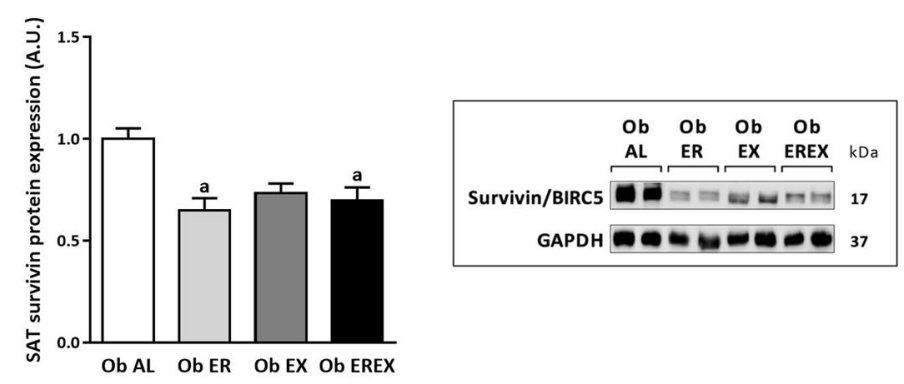

$\mathbf{F}$

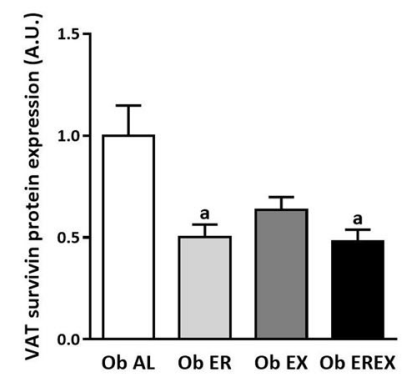

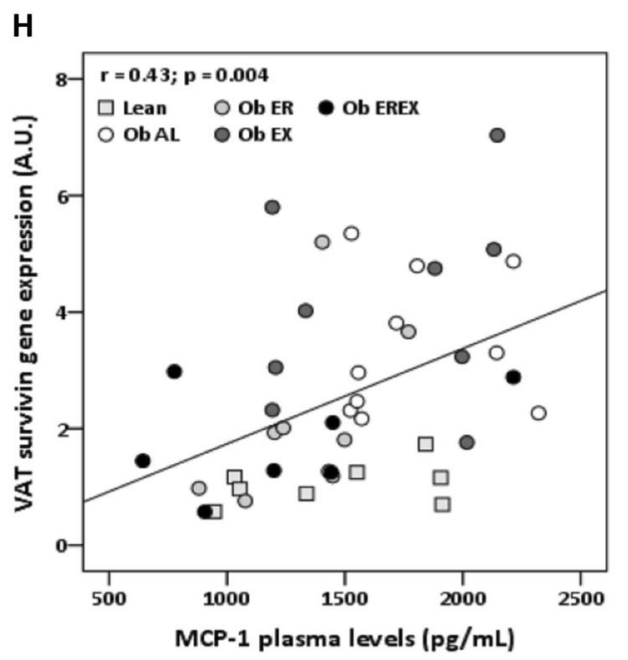

weight loss at 2 years [32], at 1 year [33], and at 6 months [34]. The expression of survivin in SAT did not change after 2 or 1 years following bariatric surgery (Fig. 2e); however, according to results of VLCKD observed, the 
Fig. 1 Survivin expression (gene and protein) in AT from obese Zucker rats after weight-loss intervention. Survivin gene expression (a) and protein levels (b) in SAT and VAT from lean and obese animals. Effect of 4 weeks of weight-loss interventions on survivin expression compared with that in the obese ad libitum group. Survivin gene expression (c) and protein levels (d) in SAT. Survivin gene expression (e) and protein levels (f) in VAT. Correlation between survivin transcript levels in VAT and FM (g), and MCP-1 (h). Data are represented as the mean $\pm \mathrm{SEM}, n=10$ animals/group. ${ }^{\mathrm{a}} P<0.05$ vs. $\mathrm{Ob} \mathrm{AL},{ }^{\mathrm{b}} P<0.05$ vs. Ob ER, and ${ }^{\mathrm{c}} P<0.05 \mathrm{Ob} \mathrm{EX}$. Ob AL were evaluated by univariant ANOVA and Bonferroni post hoc tests. $\mathrm{Ob} \mathrm{Al}$ obese ad libitum group, Ob ER obese energy restriction group, Ob EX obese exercise group, Ob EREX obese energy restriction plus exercise group.

expression of survivin in PBMCs was significantly decreased after 6 months following bariatric surgeryinduced weight loss (Fig. 2e).

\section{Discussion}

Survivin is an oncogene that was characterized as one of the most powerful inhibitors of apoptosis [35, 36]. In this study, we demonstrated that survivin expression in obesity is elevated in VAT compared with that in SAT by using animal models of both genetic and DIO, such overexpression is reversible under energy-restricting conditions. These findings were also demonstrated in blood leukocytes from patients with obesity compared with lean individuals and after weight-loss interventions with a VLCKD or bariatric surgery.

During this century, the close relationship between obesity and cancer has become evident and represents one of the greatest challenges among the pathologies associated with obesity [37]. Despite this, the mechanisms responsible for this association have not yet been characterized in detail. Previous studies have revealed that leptin levels induce carcinogenesis and this effect could be mediated in part by survivin $[24,38,39]$. Leptin upregulates survivin expression in cardiomyocytes and breast cancer cells [40, 41]. Subjects with obesity have high levels of circulating leptin unable to exert its function, a state named leptin resistance [42, 43]. Relevantly, the current study has been performed using Zucker rats, a monogenic obesity model that presents a missense mutation in the leptin receptor gene, which reduces its affinity for leptin and its capacity for signal transduction [44]. Therefore, the obesityrelated expression of survivin observed in this study may be independent of the action of leptin. The increased expression of survivin in both genetic and DIO was specially detected in VAT, while the transcript levels of survivin was similar in SAT from animals with obesity than animals with normal weight. Conversely, when the expression of survivin was evaluated in animals that were fed a HFD during a long time (29 vs. 12 weeks), the obesity-related increase in the expression of survivin was also observed in the SAT of these animals. These results could suggest that survivin is regulated by factors induced by a dysfunctional VAT, such as inflammation and oxidative stress, which were proposed as major promoters of obesity-related diseases, such as insulin resistance, cardiovascular disease, and cancer [12, 13]. So, exposing an obesogenic environment for a long time would also alter the functionality of the SAT which could secrete the same inflammatory and oxidative stress factors that regulate survivin expression in both VAT and SAT of these animals.

Weight-loss treatments considerably reduce these intrinsic pathological conditions within AT, and therefore, they also decrease the health risks [45-47]. In this context, our data clearly indicate that survivin levels in VAT and in SAT are reduced by $50 \%$ after weight loss induced by energy restriction treatment. It suggests that survivin expression is associated with adiposity features. In fact, positive correlations with BW and FM were observed in the animal model of Zucker rats. Correlations were also observed between survivin expression and plasma levels of MCP-1, a relevant marker of obesity-related inflammation.

Even though physical exercise is able to induce a reduction in several pathological conditions associated to obesity, in the current study, physical exercise did not influence the survivin expression. Further studies are need to demonstrate if the expression of survivin is not modulated by exercise or whether more time or intensity of exercise is necessary to detect these effects.

In order to translate the preclinical results to a clinical setting, in the current study, the survivin expression was also evaluated in human PBMCs, as similar of that observed in AT. Accordingly, the results observed in the animal models were replicated in PBMCs from patients with obesity compared with subjects who were normal weight. Moreover, after weight loss induced by energy-restricted diet or bariatric surgery [34] the survivin expression in blood leukocytes was downregulated and correlated with obesity features; similar to that observed in the preclinical models. However, the expression of survivin did not change in SAT from patients after bariatric surgery [32, 33]. It could suggest that the expression of survivin in PBMCs could be mirroring the expression of this gene in VAT. In fact, both, a VLCKD [31] and bariatric surgery [48] are able to induce a huge reduction in BW and FM, particularly visceral FM. These finding further corroborates the use of blood leukocytes as a noninvasive model for the molecular monitoring of the state of AT in both physiological and pathological states.

Therefore, our current study revealed an increase in the antiapoptotic pathways in AT under conditions of obesity and their correlation with the degree of systemic inflammation in animal models, highlighting that survivin could be related to the abnormal expansion of FM and the 
A

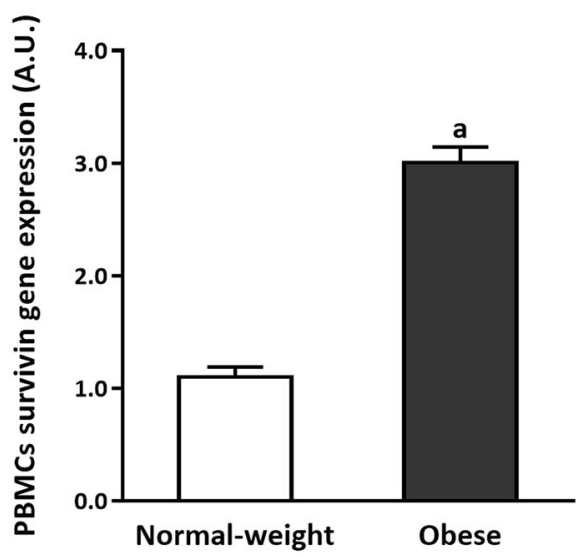

C

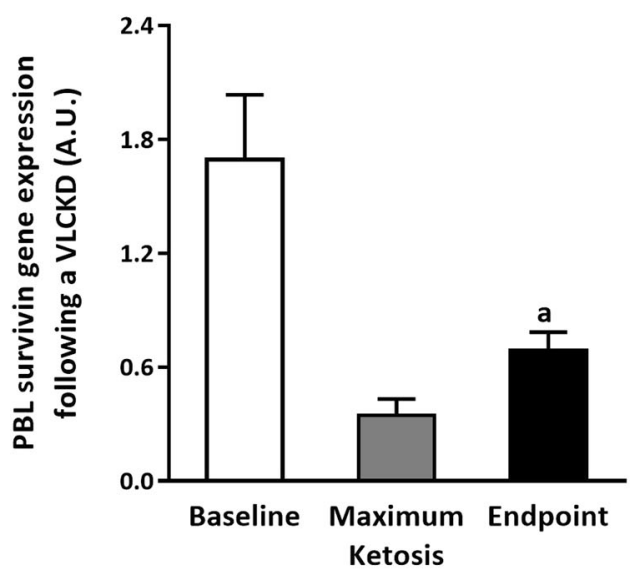

B

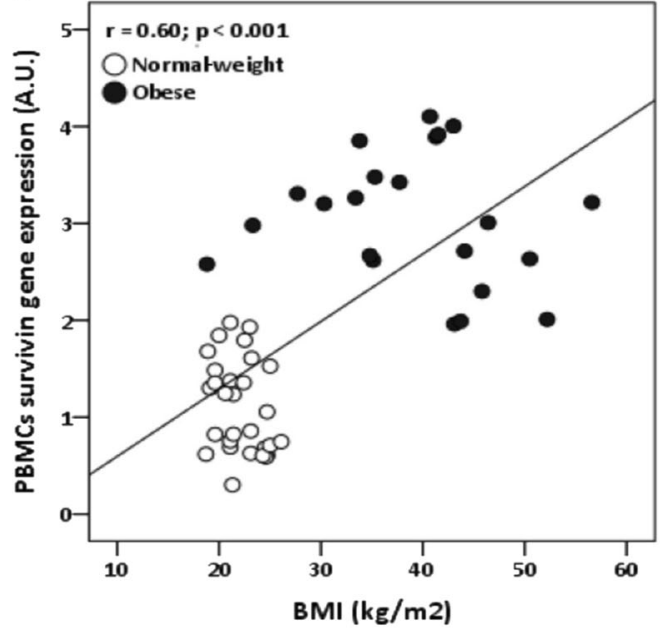

D

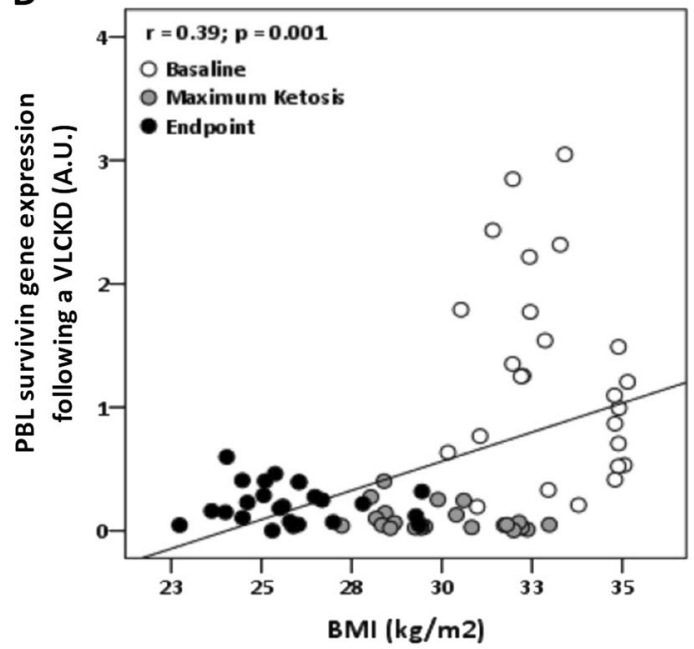

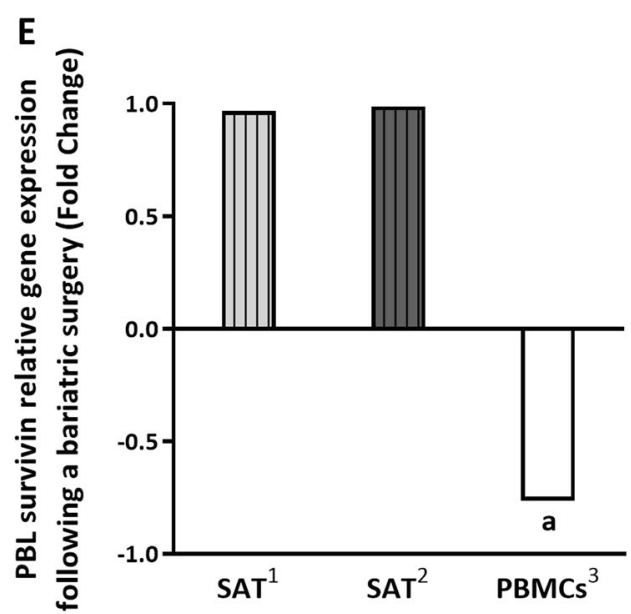

development of tumor processes. Notably, our findings also indicate that these associations between survivin expression and adiposity features are restored after weight loss.
The strength of this study is the use of different animal models of obesity and the development of an energy restriction treatment to lose weight in both genetic and DIO 
Fig. 2 Survivin gene expression in humans related with adiposity grade. a Survivin gene expression in PBMCs in normal-weight subjects compared with patients with obesity. Data are represented as the mean \pm SEM, $n=22-29$ subjects/group. ${ }^{\text {a }} P<0.05$ vs. normal weight, evaluated by Student's $t$ test. b Correlation between the survivin transcripts levels in PBMCs and BMI in a group of healthy with obesity and normal-weight patients. c Survivin gene expression in PBL in patients with obesity after VLCKD treatment. Data are represented as the mean $\pm \mathrm{SEM}, n=25$ subjects/group. ${ }^{\text {a }} P<0.05$ vs. baseline. d Correlation between the survivin transcript levels in PBLs and BMI in humans following a VLCKD. e Survivin gene expression in SAT and PBMCs from patients with obesity following bariatric surgery. Data are represented as the fold change in survivin transcript levels after bariatric surgery and were obtained from previously published studies following bariatric surgery-induced weight loss at 2 years $\left({ }^{1}\right.$ Ortega study [32]), at 1 year ( ${ }^{2}$ Dankel study [33]), and at 6 months $\left({ }^{3}\right.$ Pinhel study [34]). ${ }^{a} P<0.05$ vs. before bariatric surgery intervention.

models. Other strength is the replication of the preclinical results in a noninvasive sample from human cohorts of obesity and after weight-loss therapies such as a VLCKD or bariatric surgery. In fact, this study provides for the first time, evidence revealing that survivin is mainly overexpresses in obesity-related VAT and it can be evaluated in blood leukocytes for monitoring the evolution of obesity pathogenesis. A limitation of this study could be its observational design and the lack of VAT in the longitudinal cohort of patients after energy restriction treatment or bariatric surgery. This fact only allows proposing hypothesis and mechanisms on the potential involvement of VAT survivin expression in the association between obesity and cancer, rather than demonstrating causality; but they warrant the need for further studies to demonstrate the role of survivin expression in the pathogenesis of obesity.

In conclusion, the oncogene survivin has emerged as an important molecule in the biology of AT, as its expression levels could be involved in the development and maintenance of obesity. This molecule may also provide a potential link between obesity and cancer. Our results show that its expression is increased in obesity, and it is particularly elevated in VAT, a type of fat responsible for the secretion of cytokines and pro-inflammatory factors. Interventions for weight loss can reverse this effect, ultimately decreasing the levels to values similar to those of the control group. In the human cohorts evaluated, the same pattern of expression was observed, where they increased in obesity and decreased after weight-loss interventions. These data suggest that survivin could be used as a possible biomarker for the identification of a pro-tumorigenic microenvironment that may result from obesity and prevented after a therapy to lose weight. In addition, our findings also reinforce the idea of using minimally invasive human samples (blood/leukocytes) to monitor non-accessible tissues in the management of obesity.
Acknowledgements The authors thank Maribel Rendo and Maria Amil from the Department of Molecular and Cellular Endocrinology of the Instituto de Investigacion Sanitaria de Santiago (IDIS) for their support with research data management. This study was supported by the Centro de Investigacion Biomedica en Red de Fisiopatologia de la Obesidad y Nutricion (CIBERobn) and grants from the Instituto de Salud Carlos III-ISCIII (PI17/01287), and it was cofinanced by the European Regional Development Fund (FEDER). ABC is funded by a research contract "Miguel Servet" (CP17/00088) from the ISCIII and cofinanced by the European Regional Development Fund (FEDER).

Author contributions AGI and ABC designed the study. AGI, MCC, $\mathrm{AFQ}$, and MPP contributed to the acquisition of the data and samples from the animal models. GRC, MAMO, and AMS contributed to the acquisition of the data and samples from human. GG and DDL performed the nutrition intervention to lose weight. FJO, JMFR, CFN, $\mathrm{CBN}$, and MASP contributed to the acquisition of the data and samples from the bariatric surgery cohort. AGI and ABC performed the statistical analysis. AGI, MCC, and ABC wrote the first draft of the paper, and FFC contributed to the interpretation of data and critical revision of the paper. All authors were involved in the writing of the paper and approved the final version of this article.

\section{Compliance with ethical standards}

Conflict of interest $\mathrm{ABC}, \mathrm{FFC}$, and DDL received research grants and conference fees from Pronokal Spain. GG is medical director of Pronokal Group. The other authors declare no conflict of interest regarding the results of this study.

Publisher's note Springer Nature remains neutral with regard to jurisdictional claims in published maps and institutional affiliations.

\section{References}

1. Apovian CM. Obesity: definition, comorbidities, causes, and burden. Am J Manag Care. 2016;22:s176-85.

2. Bluher M. Obesity: global epidemiology and pathogenesis. Nat Rev Endocrinol. 2019;15:288-98.

3. NCD Risk Factor Collaboration (NCD-RisC). Worldwide trends in body-mass index, underweight, overweight, and obesity from 1975 to 2016: a pooled analysis of 2416 population-based measurement studies in 128.9 million children, adolescents, and adults. Lancet. 2017;390:2627-42.

4. NCD Risk Factor Collaboration (NCD-RisC). Worldwide trends in diabetes since 1980: a pooled analysis of 751 populationbased studies with 4.4 million participants. Lancet. 2016;387:1513-30.

5. Leitner DR, Fruhbeck G, Yumuk V, Schindler K, Micic D, Woodward E, et al. Obesity and type 2 diabetes: two diseases with a need for combined treatment strategies-EASO can lead the way. Obes Facts. 2017;10:483-92.

6. Van Gaal LF, Mertens IL, De Block CE. Mechanisms linking obesity with cardiovascular disease. Nature. 2006;444:875-80.

7. Lauby-Secretan B, Scoccianti C, Loomis D, Grosse Y, Bianchini F, Straif K, et al. Body fatness and cancer-viewpoint of the IARC working group. N Engl J Med. 2016;375:794-8.

8. Carobbio S, Pellegrinelli V, Vidal-Puig A. Adipose tissue function and expandability as determinants of lipotoxicity and the metabolic syndrome. Adv Exp Med Biol. 2017;960:161-96.

9. Bluher M. Adipose tissue dysfunction in obesity. Exp Clin Endocrinol Diabetes. 2009;117:241-50. 
10. Choe SS, Huh JY, Hwang IJ, Kim JI, Kim JB. Adipose Tissue Remodeling: Its Role in Energy Metabolism and Metabolic Disorders. Front Endocrinol. 2016;7:30.

11. Crewe C, An YA, Scherer PE. The ominous triad of adipose tissue dysfunction: inflammation, fibrosis, and impaired angiogenesis. J Clin Invest. 2017;127:74-82.

12. Juarez-Rojas JG, Torre-Villalvazo I, Medina-Urrutia AX, ReyesBarrera J, Sainz-Escarrega VH, Posadas-Romero C, et al. Participation of white adipose tissue dysfunction on circulating HDL cholesterol and HDL particle size in apparently healthy humans. Int J Obes (Lond). 2020;44:920-8.

13. Longo M, Zatterale F, Naderi J, Parrillo L, Formisano P, Raciti GA. Adipose tissue dysfunction as determinant of obesityassociated metabolic complications. Int J Mol Sci. 2019;20:2358.

14. Cabia B, Andrade S, Carreira MC, Casanueva FF, Crujeiras AB. A role for novel adipose tissue-secreted factors in obesity-related carcinogenesis. Obes Rev. 2016;17:361-76.

15. Chadid S, Kreger BE, Singer MR, Loring Bradlee M, Moore LL. Anthropometric measures of body fat and obesity-related cancer risk: sex-specific differences in Framingham Offspring Study adults. Int J Obes (Lond). 2020;44:601-8.

16. Ambrosini G, Adida C, Altieri DC. A novel anti-apoptosis gene, survivin, expressed in cancer and lymphoma. Nat Med. 1997;3:917-21.

17. Kolb R, Sutterwala FS, Zhang W. Obesity and cancer: inflammation bridges the two. Curr Opin Pharmacol. 2016;29:77-89.

18. Mita AC, Mita MM, Nawrocki ST, Giles FJ. Survivin: key regulator of mitosis and apoptosis and novel target for cancer therapeutics. Clin Cancer Res. 2008;14:5000-5.

19. Nachmias B, Ashhab Y, Ben-Yehuda D. The inhibitor of apoptosis protein family (IAPs): an emerging therapeutic target in cancer. Semin Cancer Biol. 2004;14:231-43.

20. Als AB, Dyrskjot L, von der Maase H, Koed K, Mansilla F, Toldbod HE, et al. Emmprin and survivin predict response and survival following cisplatin-containing chemotherapy in patients with advanced bladder cancer. Clin Cancer Res. 2007;13: 4407-14.

21. Derin D, Soydinc HO, Guney N, Tas F, Camlica H, Duranyildiz $\mathrm{D}$, et al. Serum levels of apoptosis biomarkers, survivin and TNFalpha in nonsmall cell lung cancer. Lung Cancer. 2008;59:240-5.

22. Zaffaroni N, Daidone MG. Survivin expression and resistance to anticancer treatments: perspectives for new therapeutic interventions. Drug Resist Updat. 2002;5:65-72.

23. Fukuda S, Pelus LM. Survivin, a cancer target with an emerging role in normal adult tissues. Mol Cancer Ther. 2006;5:1087-98.

24. Ejarque M, Ceperuelo-Mallafre V, Serena C, Pachon G, NunezAlvarez Y, Terron-Puig M, et al. Survivin, a key player in cancer progression, increases in obesity and protects adipose tissue stem cells from apoptosis. Cell Death Dis. 2017;8:e2802.

25. Ju L, Zhang X, Deng Y, Han J, Yang J, Chen S, et al. Enhanced expression of Survivin has distinct roles in adipocyte homeostasis. Cell Death Dis. 2017;8:e2533.

26. Crujeiras AB, Cabia B, Carreira MC, Amil M, Cueva J, Andrade $\mathrm{S}$, et al. Secreted factors derived from obese visceral adipose tissue regulate the expression of breast malignant transformation genes. Int J Obes. 2016;40:514-23.

27. Izquierdo AG, Carreira MC, Amil M, Mosteiro CS, GarciaCaballero T, Fernandez-Quintela A, et al. An energy restrictionbased weight loss intervention is able to reverse the effects of obesity on the expression of liver tumor-promoting genes. FASEB J. 2019;34:2312-25.

28. Trimboli P, Castellana M, Bellido D, Casanueva FF. Confusion in the nomenclature of ketogenic diets blurs evidence. Rev Endocr Metab Disord. 2020;21:1-3.

29. Heiker JT, Kunath A, Kosacka J, Flehmig G, Knigge A, Kern M, et al. Identification of genetic loci associated with different responses to high-fat diet-induced obesity in C57BL/6N and C57BL/6J substrains. Physiol Genomics. 2014;46:377-84.

30. de Luis D, Domingo JC, Izaola O, Casanueva FF, Bellido D, Sajoux I. Effect of DHA supplementation in a very low-calorie ketogenic diet in the treatment of obesity: a randomized clinical trial. Endocrine. 2016;54:111-22.

31. Gomez-Arbelaez D, Bellido D, Castro AI, Ordonez-Mayan L, Carreira J, Galban C, et al. Body composition changes after verylow-calorie ketogenic diet in obesity evaluated by 3 standardized methods. J Clin Endocrinol Metab. 2017;102:488-98.

32. Ortega FJ, Mercader JM, Moreno-Navarrete JM, Nonell L, Puigdecanet E, Rodriquez-Hermosa JI, et al. Surgery-induced weight loss is associated with the downregulation of genes targeted by MicroRNAs in adipose tissue. J Clin Endocrinol Metab. 2015;100:E1467-76.

33. Dankel SN, Fadnes DJ, Stavrum AK, Stansberg C, Holdhus R, Hoang T, et al. Switch from stress response to homeobox transcription factors in adipose tissue after profound fat loss. PLoS ONE. 2010;5:e11033.

34. Pinhel MAS, Noronha NY, Nicoletti CF, de Oliveira BAP, Cortes-Oliveira C, Pinhanelli VC, et al. Changes in global transcriptional profiling of women following obesity surgery bypass. Obes Surg. 2018;28:176-86.

35. Reichert S, Rodel C, Mirsch J, Harter PN, Tomicic MT, Mittelbronn M, et al. Survivin inhibition and DNA double-strand break repair: a molecular mechanism to overcome radioresistance in glioblastoma. Radiother Oncol. 2011;101:51-8.

36. Vequaud E, Desplanques G, Jezequel P, Juin P, Barille-Nion S. Survivin contributes to DNA repair by homologous recombination in breast cancer cells. Breast Cancer Res Treat. 2016;155:53-63.

37. Avgerinos KI, Spyrou N, Mantzoros CS, Dalamaga M. Obesity and cancer risk: emerging biological mechanisms and perspectives. Metabolism. 2019;92:121-35.

38. Knight BB, Oprea-Ilies GM, Nagalingam A, Yang L, Cohen C, Saxena NK, et al. Survivin upregulation, dependent on leptinEGFR-Notch1 axis, is essential for leptin-induced migration of breast carcinoma cells. Endocr Relat Cancer. 2011;18:413-28.

39. Palianopoulou M, Papanikolaou V, Stefanou N, Tsezou A. The activation of leptin-mediated survivin is limited by the inducible suppressor SOCS-3 in MCF-7 cells. Exp Biol Med. 2011;236:70-6.

40. Jiang H, Yu J, Guo H, Song H, Chen S. Upregulation of survivin by leptin/STAT3 signaling in MCF-7 cells. Biochem Biophys Res Commun. 2008;368:1-5.

41. McGaffin KR, Zou B, McTiernan CF, O’Donnell CP. Leptin attenuates cardiac apoptosis after chronic ischaemic injury. Cardiovasc Res. 2009;83:313-24.

42. Crujeiras AB, Carreira MC, Cabia B, Andrade S, Amil M, Casanueva FF. Leptin resistance in obesity: an epigenetic landscape. Life Sci. 2015;140:57-63.

43. Izquierdo AG, Crujeiras AB, Casanueva FF, Carreira MC. Leptin, obesity, and leptin resistance: where are we 25 years later? Nutrients. 2019;11:2704.

44. Chua SC Jr., White DW, Wu-Peng XS, Liu SM, Okada N, Kershaw EE, et al. Phenotype of fatty due to Gln269Pro mutation in the leptin receptor (Lepr). Diabetes. 1996;45:1141-3.

45. Crujeiras AB, Gomez-Arbelaez D, Zulet MA, Carreira MC, Sajoux I, de Luis D, et al. Plasma FGF21 levels in obese patients undergoing energy-restricted diets or bariatric surgery: a marker of metabolic stress? Int J Obes. 2017;41:1570-8.

46. Lopez-Domenech S, Martinez-Herrera M, Abad-Jimenez Z, Morillas C, Escribano-Lopez I, Diaz-Morales N, et al. Dietary weight loss intervention improves subclinical atherosclerosis and oxidative stress markers in leukocytes of obese humans. Int $\mathbf{J}$ Obes. 2019;43:2200-9. 
47. Carreira MC, Izquierdo AG, Amil M, Casanueva FF, Crujeiras AB. Oxidative stress induced by excess of adiposity is related to a downregulation of hepatic SIRT6 expression in obese individuals. Oxid Med Cell Longev. 2018;2018:6256052.
48. Merlotti C, Ceriani V, Morabito A, Pontiroli AE. Subcutaneous fat loss is greater than visceral fat loss with diet and exercise, weightloss promoting drugs and bariatric surgery: a critical review and meta-analysis. Int J Obes. 2017;41:672-82.

\section{Affiliations}

Andrea G. Izquierdo $\mathbb{1}^{1,2} \cdot \operatorname{Marcos}$ C. Carreira ${ }^{2,3} \cdot$ Gemma Rodriguez-Carnero $^{1} \cdot$ Alfredo Fernandez-Quintela $^{2,4}$. Aurelio M. Sueiro ${ }^{3}$ - Miguel A. Martinez-Olmos ${ }^{1,2} \cdot$ German Guzman $^{5} \cdot$ Daniel De Luis $^{6}{ }^{6} \cdot$ Marcela A. S. Pinhel $^{7}$. Carolina F. Nicoletti ${ }^{7}$. Carla B. Nonino ${ }^{7}$ - Francisco J. Ortega $\mathbb{B}^{2,8} \cdot$ Maria P. Portillo $^{2,4} \cdot$ Jose M. Fernandez-Real $^{2,8}$. Felipe F. Casanueva ${ }^{2,3} \cdot$ Ana B. Crujeiras $\mathbb{1}^{1,2}$

1 Epigenomics in Endocrinology and Nutrition Group, Instituto de Investigacion Sanitaria (IDIS), Complejo Hospitalario Universitario de Santiago (CHUS/SERGAS), Santiago de Compostela, Spain

2 CIBER Fisiopatologia de la Obesidad y Nutricion (CIBERobn), Madrid, Spain

3 Molecular Endocrinology Group, Instituto de Investigacion Sanitaria (IDIS), Complejo Hospitalario Universitario de Santiago (CHUS/SERGAS) and Santiago de Compostela University (USC), Santiago de Compostela, Spain

4 Nutrition and Obesity Group, Department of Nutrition and Food Science, University of the Basque Country (UPV/EHU) and Lucio Lascaray Research Institute, Vitoria, Spain
5 Medical Department Pronokal, Pronokal Group, Barcelona, Spain

6 Endocrinology and Nutrition Research Center, School of Medicine, Department of Endocrinology and Nutrition, Hospital Clinico Universitario, University of Valladolid, Valladolid, Spain

7 Department of Internal Medicine, Laboratory of Nutrigenomic Studies, Ribeirao Preto Medical School, FMRP, University of Sao Paulo, USP, Sao Paulo, Brazil

8 Department of Diabetes, Endocrinology, and Nutrition (UDEN), Institut d'Investigació Biomèdica de Girona (IdIBGi), Girona, Spain 